\title{
A Novel Analytical Approach for the Solution of Fractional-Order Diffusion-Wave Equations
}

\author{
Saima Mustafa ${ }^{1} \mathbb{(}$, Hajira $^{2}$, Hassan Khan ${ }^{2,3, *}$, Rasool Shah ${ }^{2} \mathbb{E}$ and Saadia Masood ${ }^{1}$ \\ 1 Department of Mathematics, Pir Mehr Ali Shah Arid Agriculture University, Rawalpindi 46000, Pakistan; \\ saimamustafa28@gmail.com (S.M.); saadia.masood@uaar.edu.pk (S.M.) \\ 2 Department of Mathematics, Abdul Wali Khan University Mardan, Mardan 23200, Pakistan; \\ hajira@awkum.edu.pk (H.); rasoolshah@awkum.edu.pk (R.S.) \\ 3 Depatment of Mathematics, Near East University TRNC, Mersin 10, Nicosia 99138, Turkey \\ * Correspondence: hassankhan@awkum.edu.pk
}

check for updates

Citation: Mustafa, S.; Hajira; Khan,

H.; Shah, R.; Masood, S. A Novel

Analytical Approach for the Solution of Fractional-Order Diffusion-Wave

Equations. Fractal Fract. 2021, 5, 206. https://doi.org/10.3390/

fractalfract5040206

Academic Editor: David Kubanek

Received: 27 September 2021

Accepted: 6 November 2021

Published: 11 November 2021

Publisher's Note: MDPI stays neutral with regard to jurisdictional claims in published maps and institutional affiliations.

Copyright: (c) 2021 by the authors. Licensee MDPI, Basel, Switzerland. This article is an open access article distributed under the terms and conditions of the Creative Commons Attribution (CC BY) license (https:// creativecommons.org/licenses/by/ $4.0 /)$.

\begin{abstract}
In the present note, a new modification of the Adomian decomposition method is developed for the solution of fractional-order diffusion-wave equations with initial and boundary value Problems. The derivatives are described in the Caputo sense. The generalized formulation of the present technique is discussed to provide an easy way of understanding. In this context, some numerical examples of fractional-order diffusion-wave equations are solved by the suggested technique. It is investigated that the solution of fractional-order diffusion-wave equations can easily be handled by using the present technique. Moreover, a graphical representation was made for the solution of three illustrative examples. The solution-graphs are presented for integer and fractional order problems. It was found that the derived and exact results are in good agreement of integer-order problems. The convergence of fractional-order solution is the focus point of the present research work. The discussed technique is considered to be the best tool for the solution of fractional-order initial-boundary value problems in science and engineering.
\end{abstract}

Keywords: Adomian decomposition method; Caputo derivative; initial-boundary value problems; fractional diffusion-wave equations

\section{Introduction}

The extension of the classical calculus is known as Fractional Calculus (FC). Many physical phenomena are described more accurately by FC. The dynamics of real world complicated problems are mostly related with FC. Due to the non-local nature of fractional operators several natural phenomena are described in a systematic and better way by them. In this regard, FC plays a very important role to model the frequency dependent damping behavior of many viscoelastic material, substrates and interfaces between nanoparticles. The subject of FC has also an important contribution in other branches of applied sciences, such as electromagnetic, cosmology, electrochemistry, edge detection, material science, signal processing, viscoelasticity, traffic system, telecommunication, genetics algorithm, robotic technology, acoustics, economy and physics [1,2].

Fractional-order partial differential equations (FPDEs) are the generalization of the classical integer order partial differential equations (PDEs). Fractional approach is now considered to be the most powerful tool for modeling and applied in wave propagation, anomalous diffusion tools, turbulence and mechanics [1,3,4]. There are many applications of FPDES in biomedical engineering, finance, probability theory and hydrology. Recently, FPDES are used as an effective tool for modeling of various natural processes such as, electrochemistry, computational biology, physics, fluid mechanics and many other fields [5-9]. FPDEs are applied as a major tool for the description of natural processes and are more appropriate than the ordinary (PDEs) in many ways, some of them are quasi chaotic dynamical system, the dynamic of complex material and random walk with memory [10-13]. 
In addition, superdiffusion and subdiffusion are the processes described more effectively by FPDEs [14]. Most of the important phenomena including fluid dynamics, polarization, electrical processes, economics, colored noise, electrochemical processes, statistical mechanics and traffic flow are accurately described by FPDEs [8,14-18].

The numerical and analytical solutions of FPDEs are considered to be a hard area of research and therefore the researchers have given full attention to develop strong and novel techniques to solve FPDEs. Some of the novel applied methods are generalized differential transform method [19], Chebyshev wavelet method [20], meshless method [21,22], finite volume method $[23,24]$, fractional difference method $[25,26]$, homotopy analysis method [27], reduced differential transform method [28], spectral method [29], the fractional sub-equation method [30], the exponential function method [31] and analytical solution in term of Mittag-Leffler function [32].

The fractional-order diffusion-wave equations (FDWEs) are the most important type of anomalous diffusion equations deduced from the classical diffusion-wave equations [33]. The fractional-diffusion equations (FDEs) were introduced by Nigmatullin [34] in physics to describe the special type of porous media. He discovered that some of the electromagnetic, mechanical and acoustic can be modeled more properly with the help of FDWEs. The FDEs are solved by Mark M. Meerschaert et al. in [35]. Agarwal [36] applied the method of separation of variables to the FDWEs to identify the eigenfunctions. Gorenflo et al. [37] described the scale invariant solution for FDWEs. Agarwal has modified this procedure to the DWEs containing fourth-order derivative term [38]. The mean-square solution containing Green function and spectral representation of FDWEs are given by Anh and Leonenko [39]. Luckho [40-42] has discussed the solution of FDWEs within time and space variable. Kenichi Sakamoto et al. solved FDWEs in [43]. For the existence and uniqueness of the solutions see [44].

In this paper we will work on the solution of FDWEs by applying a new technique of Adomian decomposition method (ADM). ADM along with its modifications are used to solve initial and boundary value problems. The proposed technique gives the solution in a finite series form, which is easily computable. Several modifications to ADM are done by many researchers to increase the accuracy of the method [45-48]. Other modifications can be seen in [49-55]. Momani used ADM to solve Navier's stoke equations of one-dimension [56]. The ADM has been used to solve FPDEs [57,58], integraldifferential equations [59,60], differential-difference equations [61,62], differential algebraic equations $[63,64]$ and vice versa.

In our work, we will use a new technique of ADM for the solution of FDWEs along with initial and boundary conditions, as used by Elaf Jaafer Ali in [65]. He also applied the same procedure for the fourth order parabolic PDEs in combination with variation iteration method in [66,67]. The same procedure is applied to the problems in [68] with the homotopy perturbation method by Elaf Jaafer Ali and the results are excellent. The one thing is common in his work that he used the procedure for initial-boundary problems. The applied procedure provides better accuracy, because of using new initial approximation in each iteration. For justification some examples are discussed in this paper.

\section{Preliminaries}

In this section, a few definitions related to our work take into consideration.

Definition 1. The integral operator of Reimann-Liouville (RL) having arbitrary order $\alpha(\alpha \geq 0)$ of a function $u(t)$ is given by [69]

$$
j^{\alpha} u(t)=\frac{1}{\Gamma(\alpha)} \int_{0}^{t}(t-\delta)^{\alpha-1} u(\delta) d \delta,
$$


where, $\Gamma(\alpha)=\int_{0}^{\infty} t^{\alpha-1} e^{-t} d t$ is the gamma function which provides the converges of Equation (1) on $(0, \infty)$ point wise. The following relations are satisfied by the $R L$ integral operator.

$$
\left\{\begin{array}{l}
j^{\alpha} j^{q} u(t)=j^{q} j^{\alpha} u(t) \\
j^{\alpha} j^{q} u(t)=j^{\alpha+q} u(t) \\
j^{\alpha} t^{q}=\frac{\Gamma(q+1)}{\Gamma(q+\alpha+1)} t^{\alpha+q}
\end{array}\right.
$$

Definition 2. For a function $u(t)$ Caputo operator of fractional order $\alpha$ is given as,

$$
D^{\alpha} u(t)=\frac{1}{\Gamma(n-\alpha)} \int_{0}^{t} \frac{u^{(n)}(t)}{(t-\tau)^{\alpha+1-n}} d \tau \quad n-1<\alpha<n, n=[\alpha]+1,
$$

such that Equation (2) satisfies the following properties.

$$
D^{\alpha} t^{v}=\left\{\begin{array}{l}
0, v \in N, v<[\alpha], \\
\frac{\Gamma(v+1)}{1+v-\alpha} t^{v-\alpha} .
\end{array}\right.
$$

Definition 3. The Mittag-Leffler function ([69]) is

$$
E_{\alpha}(z)=\sum_{n=0}^{\infty}\left(\frac{z^{n}}{\Gamma(n \alpha+1)}\right), \quad \alpha>0, \quad z \in C .
$$

\section{ADM Implementation}

This method was discovered by Adomian in (1994) to solve differential and integrodifferential equations. The present method can be explained by using the following procedure. Let

$$
F(u(x))=g(x),
$$

where, $g(x)$ is the known function, $F$ is differential operator, which can be decomposed as follows.

$$
F(u)=L u+R u+N u,
$$

where $L$ is the invertible operator of highest derivative and $R$ and $N$ are linear and nonlinear terms, respectively. Then Equation (3) has the representation as follows

$$
L u+R u+N u=g .
$$

Taking $L^{-1}$ of Equation (5), we get

$$
u=\eta+L^{-1}(g)-L^{-1}(R u)-L^{-1}(N u),
$$

where, $\eta$ is the constant of integration. The ADM solution can be represented in the form of infinite series as,

$$
u=\sum_{n=0}^{\infty} u_{n} .
$$

The non-linear term $N u$ is denoted by $A_{n}$ and is defined as,

$$
N u=\sum_{n=0}^{\infty} A_{n},
$$

with the help of the following formula, we can calculate $A_{n}$ as,

$$
A_{n}=\frac{1}{n !} \frac{d^{n}}{d \lambda^{n}} N\left(\sum_{k=0}^{\infty}\left(\lambda^{k} u_{k}\right)\right), \quad n=0,1, \ldots
$$


The following relation is obtained to represent the solution of Equation (3),

$$
\left\{\begin{array}{l}
u_{0}=\eta+L^{-1}(g), \quad n=0, \\
u_{n+1}=L^{-1}\left(R u_{n}\right)-L^{-1}\left(A_{n}\right) . \quad n \geq 0
\end{array}\right.
$$

\section{New Idea Based on ADM}

To understand the present procedure, the following one dimensional equation is considered with initial and boundary conditions.

$$
D^{\alpha}(u(\xi, \tau))=D_{\xi \xi} u(\xi, \tau)+R(\xi, \tau), \quad 0<\xi<1, \tau>0,1<\alpha \leq 2,
$$

with the following initial and boundary conditions as,

$$
\begin{cases}u(\xi, \tau)=f_{0}(\xi), & \frac{\partial u(\xi, 0)}{\partial \tau}=f_{1}(\xi), \quad 0 \leq \xi \leq 1, \\ u(0, \tau)=g_{0}(\tau), & u(1, \tau)=g_{1}(\tau), \quad \tau>0 .\end{cases}
$$

where, $R(\xi, \tau)$ is the source term. The new solution $\left(u_{n}^{*}\right)$ is then calculated for using the new proposed technique.

$$
u_{n}^{*}=u_{n}(\xi, \tau)+(1-\xi)\left[g_{0}(\tau)-u_{n}(0, \tau)\right]+\xi\left[g_{1}(\tau)-u_{n}(1, \tau)\right],
$$

using ADM the operator form of Equation (21) is

$$
L u=D_{\xi \xi} u(\xi, \tau)+R(\xi, \tau),
$$

where $L$ is defined as,

$$
L=\frac{\partial^{\alpha}}{\partial \tau^{\alpha}}, \quad 1<\alpha \leq 2
$$

Hence $L^{-1}$ is defined as,

$$
L^{-1}(.)=I^{\alpha}(.) d \tau,
$$

Applying $L^{-1}$ to Equation (8), we have

$$
u(\xi, \tau)=+L^{-1}\left(D_{\xi \xi} u(\xi, \tau)+R(\xi, \tau)\right),
$$

using ADM the initial approximation becomes

$$
u_{0}(\xi, \tau)=u(\xi, 0)+\tau\left(\partial_{t} u(\xi, 0)\right)+L^{-1}(R(\xi, \tau)) .
$$

And use new technique of ADM the iteration formula becomes

$$
u_{n+1}(\xi, \tau)=\left(D_{\xi \xi} u_{n}^{*}\right) . \quad n=0,1, \ldots
$$

It is obvious that new solution $u_{n}^{*}$ of Equation (21) satisfy both the initial and boundary conditions,

$$
\text { at } \begin{aligned}
\tau=0, & u_{n}^{*}(\xi, 0)=u_{n}(\xi, 0), \\
\xi=0, & u_{n}^{*}(0, \tau)=g_{0}(\tau), \\
\xi=1, & u_{n}^{*}(1, \tau)=g_{1}(\tau) .
\end{aligned}
$$

It is shown that the proposed technique works effectively for the solution of FPDEs with initial and boundary conditions.

\section{Numerical Results}

In this section, we will present the solution of some illustrative examples by using the new technique based on ADM. 


\subsection{Example 1}

Consider FDWE of the form [70]

$$
D_{\tau}^{\alpha} u(\xi, \tau)=u_{\xi \xi}(\xi, \tau)+\sin (\pi \xi), \quad 0<\xi<1,1<\alpha \leq 2,
$$

with the following initial and boundary conditions,

$$
\begin{aligned}
& u(\xi, 0)=0, \quad \partial_{t} u(\xi, 0)=0, \quad 0 \leq \xi \leq 1, \\
& u(0, \tau)=u(1, \tau)=0, \quad 0 \leq \tau \leq 1 .
\end{aligned}
$$

The problem has the exact solution at $\alpha=2$ as,

$$
u(\xi, \tau)=\frac{1}{\pi^{2}}\left[1-E_{\alpha}\left(-\pi^{2} \tau^{\alpha}\right)\right] \sin (\pi \xi),
$$

where

$$
E_{\alpha}=\sum_{k=0}^{\infty} \frac{z^{k}}{\Gamma(\alpha k+1)}
$$

is an one-parameter Mittag-Leffler function.

Applying the new technique based on ADM to Equation (11), we get the following result.

$$
u_{n}^{*}(\xi, \tau)=u_{n}(\xi, \tau)+(1-\xi)\left[0-u_{n}(0, \tau)\right]+\xi\left[0-u_{n}(1, \tau)\right],
$$

where $n=0,1, \ldots$ Equation (11) can be written as,

$$
L u=u_{\xi \xi}(\xi, \tau)+\sin (\pi \xi),
$$

where

$$
L=D_{\tau}^{\alpha}, \quad 1<\alpha \leq 2 .
$$

Using $L^{-1}$ Equation (13) can be written as,

$$
u(\xi, \tau)=u(\xi, 0)+\tau\left(\partial_{t} u(\xi, 0)\right)+L^{-1}\left(u_{\xi \xi}(\xi, \tau)+\sin (\pi \xi)\right) .
$$

Using ADM procedure, the initial approximation becomes,

$$
\begin{gathered}
u_{0}(\xi, \tau)=u(\xi, 0)+\tau\left(\partial_{t} u(\xi, 0)\right)+L^{-1}(\sin (\pi \xi)), \\
=0+0+\frac{\tau^{\alpha} \sin (\pi \xi)}{\Gamma(\alpha+1)} \\
u_{0}(\xi, \tau)=\frac{\tau^{\alpha} \sin (\pi \xi)}{\Gamma(\alpha+1)} .
\end{gathered}
$$

And use the new technique of initial approximation $u_{n}^{*}$ the iteration formula becomes

$$
u_{n+1}(\xi, \tau)=L^{-1}\left(\frac{\partial^{2} u_{n}^{*}}{\partial \xi^{2}}\right)
$$

By putting initial and boundary conditions in Equation (12), for $n=0$

$$
\begin{gathered}
u_{0}^{*}(\xi, \tau)=u_{0}(\xi, \tau)+(1-\xi)\left[0-u_{0}(0, \tau)\right]+\xi\left[0-u_{0}(1, \tau)\right], \\
=\frac{\tau^{\alpha}}{\Gamma(\alpha+1)}(\sin (\pi \xi))+(1-\xi)[0-0]+\xi[0-0], \\
u_{0}^{*}(\xi, \tau)=\frac{\tau^{\alpha} \sin (\pi \xi)}{\Gamma(\alpha+1)} .
\end{gathered}
$$


From Equation (15), we have

$$
\begin{gathered}
u_{1}(\xi, \tau)=L^{-1}\left(\frac{\partial^{2} u_{0}^{*}}{\partial \xi^{2}}\right)=L^{-1}\left(\frac{-\tau^{\alpha} \pi^{2} \sin (\pi \xi)}{\Gamma(\alpha+1)}\right) \\
u_{1}(\xi, \tau)=\frac{-\tau^{2 \alpha} \pi^{2} \sin (\pi \xi)}{\Gamma(\alpha+1)} .
\end{gathered}
$$

For $n=1$ Equation (12) becomes,

$$
\begin{gathered}
u_{1}^{*}(\xi, \tau)=u_{1}(\xi, \tau)+(1-\xi)\left[0-u_{1}(0, \tau)\right]+\xi\left[0-u_{1}(1, \tau)\right], \\
=\frac{-\tau^{2 \delta} \pi^{2} \sin (\pi \xi)}{\Gamma(2 \alpha+1)}+(1-\xi)[0-0]+\xi[0-0], \\
u_{1}^{*}(\xi, \tau)=\frac{-\tau^{2 \alpha} \pi^{2} \sin (\pi \xi)}{\Gamma(2 \alpha+1)} .
\end{gathered}
$$

From Equation (15), we have

$$
\begin{gathered}
u_{2}(\xi, \tau)=L^{-1}\left(\frac{\partial^{2} u_{1}^{*}}{\partial \xi^{2}}\right)=L^{-1}\left(\frac{\tau^{2 \alpha} \pi^{4} \sin (\pi \xi)}{\Gamma(2 \alpha+1)}\right) \\
u_{2}(\xi, \tau)=\frac{\tau^{3 \alpha} \pi^{4} \sin (\pi \xi)}{\Gamma(3 \alpha+1)} .
\end{gathered}
$$

For $n=2$ Equation (12), becomes

$$
\begin{gathered}
u_{2}^{*}(\xi, \tau)=u_{2}(\xi, \tau)+(1-\xi)\left[0-u_{2}(0, \tau)\right]+\xi\left[0-u_{2}(1, \tau)\right], \\
=\frac{\tau^{3 \alpha} \pi^{4} \sin (\pi \xi)}{\Gamma(3 \alpha+1)}+(1-\xi)[0-0]+\xi[0-0], \\
u_{2}^{*}(\xi, \tau)=\frac{\tau^{3 \alpha} \pi^{4} \sin (\pi \xi)}{\Gamma(3 \alpha+1)} .
\end{gathered}
$$

From Equation (15), we have

$$
\begin{gathered}
u_{3}(\xi, \tau)=L^{-1}\left(\frac{\partial^{2} u_{2}^{*}}{\partial \xi^{2}}\right)=L^{-1}\left(\frac{-\tau^{3 \alpha} \pi^{6} \sin (\pi \xi)}{\Gamma(3 \alpha+1)}\right), \\
u_{3}(\xi, \tau)=\frac{-\tau^{4 \alpha} \pi^{6} \sin (\pi \xi)}{\Gamma(4 \alpha+1)} .
\end{gathered}
$$

Thus the ADM solution in the series form is,

$$
\begin{gathered}
u(\xi, \tau)=u_{0}(\xi, \tau)+u_{1}(\xi, \tau)+u_{2}(\xi, \tau)+u_{3}(\xi, \tau)+\ldots \\
=\frac{\tau^{\alpha} \sin (\pi \xi)}{\Gamma(\alpha+1)}-\frac{\tau^{2 \alpha} \pi^{2} \sin (\pi \xi)}{\Gamma(2 \alpha+1)}+\frac{\tau^{3 \alpha} \pi^{4} \sin (\pi \xi)}{\Gamma(3 \alpha+1)}-\frac{\tau^{4 \alpha} \pi^{6} \sin (\pi \xi)}{\Gamma(4 \alpha+1)}+\ldots
\end{gathered}
$$

In Figure $1 \mathrm{a}, \mathrm{b}$, the obtained solutions of Example 1 are plotted at various fractionalorders of the derivatives, Figure 1a,b provides the 2D and 3D, ADM solutions graphs and confirmed the closed contact with the exact solution of Example 1. Similarly, in Figure 2, the closed contact between the exact and obtained solutions of Example 1 at integer order 
of the derivative is investigated. From the graphs, it is confirmed that the exact and derived results are in closed contact with each other. Thus the proposed method has provided an accurate solution for Example 1.

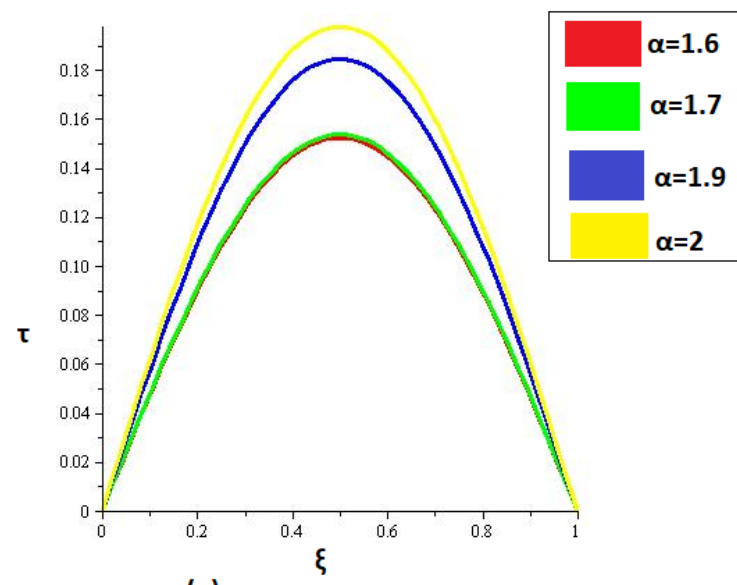

(a)

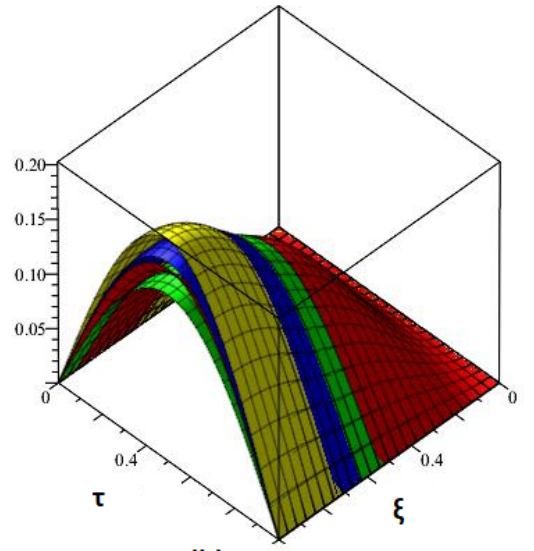

(b)

Figure 1. ADM solution plots (a) 2D and (b) 3D of Example 1 at different $\alpha$.
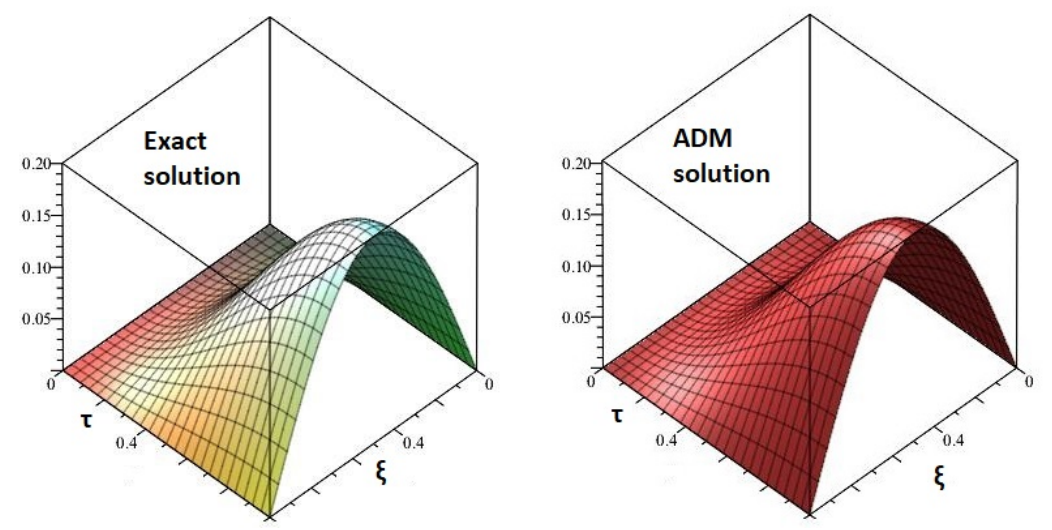

Figure 2. Exact and ADM solution 3D plots of Example 1 at $\alpha=2$.

Figure 3 is plotted to confirm the closed contact between the exact and derived solution of Example 1 in 2D-space. Figure 3 represents the Exact and ADM solutions, respectively, at $\alpha=2$.
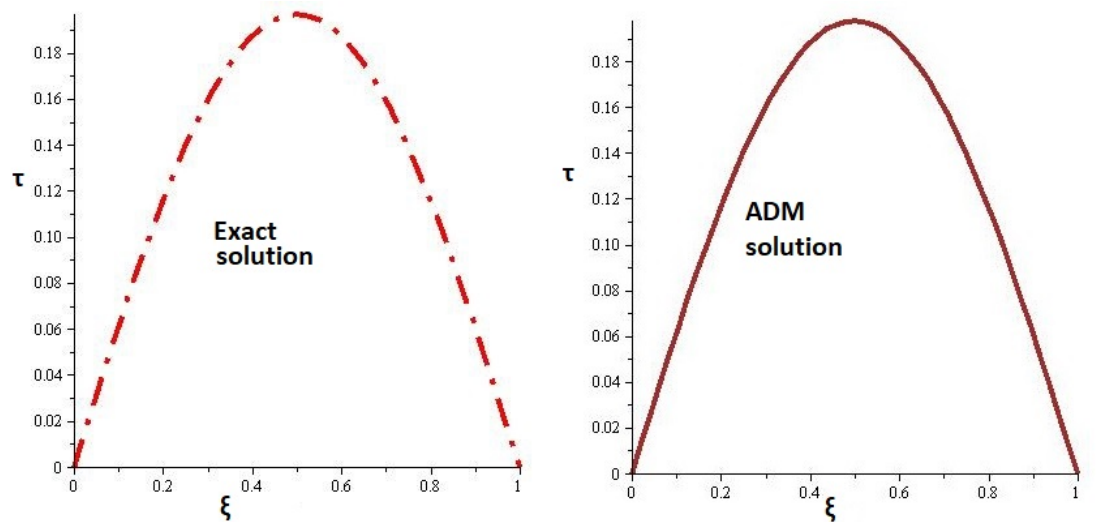

Figure 3. Exact and ADM solution 2D plots of Example 1 at $\alpha=2$. 


\subsection{Example 2}

Consider the FWDE of the form [70]

$$
D_{\tau}^{\alpha} u(\xi, \tau)=u_{\xi \xi}(\xi, \tau)+e^{\xi}\left[\Gamma(1+\alpha)-\tau^{\alpha}-\tau-1\right], \quad 0<\xi<1,0<\tau \leq 1 .
$$

With the following initial and boundary conditions,

$$
\begin{aligned}
& u(\xi, 0)=e^{\xi}, \partial_{t} u(\xi, 0)=e^{\xi} \quad 0<\xi<1, \\
& u(0, \tau)=\tau^{\alpha}+\tau+1, u(1, \tau)=e^{1}\left[\tau^{\alpha}+\tau+1\right], 0<\tau \leq 1 .
\end{aligned}
$$

The problem has the Exact solution at $\alpha=2$ as follows,

$$
u(\xi, \tau)=e^{\xi}\left[\tau^{\alpha}+\tau+1\right] .
$$

Applying the new technique based on ADM to Equation (16), we get the following result.

$$
u_{n}^{*}(\xi, \tau)=u_{n}(\xi, \tau)+(1-\xi)\left[0-u_{n}(0, \tau)\right]+\xi\left[0-u_{n}(1, \tau)\right] .
$$

where $n=0,1, \ldots$

Equation (16), can be written as,

$$
L u=u_{\xi \xi}(\xi, \tau)+e^{\xi}\left[\Gamma(1+\alpha)-\tau^{\alpha}-\tau-1\right],
$$

where $L=D_{\tau}^{\alpha}$ and $L^{-1}$ is defined as,

$$
L^{-1}(.)=I^{\alpha}(.) d \tau
$$

Taking Equation $L^{-1}$ on both sides of Equation (18), we get,

$$
u(\xi, \tau)=u(\xi, 0)+\tau\left(\partial_{t} u(\xi, 0)\right)+L^{-1}\left(u_{\xi \xi}(\xi, \tau)+e^{\xi}\left[\Gamma(1+\alpha)-\tau^{\alpha}-\tau-1\right]\right) .
$$

Using ADM solution, the initial approximation becomes,

$$
\begin{gathered}
u_{0}(\xi, \tau)=u(\xi, 0)+\tau\left(\partial_{t} u(\xi, 0)\right)+L^{-1}\left(e^{\xi}\left[\Gamma(1+\alpha)-\tau^{\alpha}-\tau-1\right]\right), \\
u_{0}(\xi, \tau)=e^{\xi}+\tau\left(e^{\xi}\right)+e^{\xi}\left(\tau^{\alpha}-\frac{\tau^{2 \alpha} \Gamma(\alpha+1)}{\Gamma(2 \alpha+1)}-\frac{\tau^{\alpha+1}}{\Gamma(\alpha+2)}-\frac{\tau^{\alpha}}{\Gamma(\alpha+1)}\right),
\end{gathered}
$$

and use the new technique of initial approximation $u_{n}^{*}$ the iteration formula becomes

$$
u_{n+1}(\xi, \tau)=L^{-1}\left(\frac{\partial^{2} u_{n}^{*}}{\partial \xi^{2}}\right)
$$

By putting the initial and boundary condition in Equation (17), for $n=0$

$$
u_{0}^{*}(\xi, \tau)=u_{0}(\xi, \tau)+(1-\xi)\left[0-u_{0}(0, \tau)\right]+\xi\left[0-u_{0}(1, \tau)\right],
$$

Let

$$
\begin{array}{r}
u_{0}(\xi, \tau)=e^{\xi}+\tau\left(e^{\xi}\right)+e^{\xi}\left(\tau^{\alpha}-\frac{\tau^{2 \alpha} \Gamma(\alpha+1)}{\Gamma(2 \alpha+1)}-\frac{\tau^{\alpha+1}}{\Gamma(\alpha+2)}-\frac{\tau^{\alpha}}{\Gamma(\alpha+1)}\right) \\
(1-\xi)\left[0-u_{0}(0, \tau)\right]=(1-\xi)\left[\tau^{\alpha}+\tau+1-1-\tau-\tau^{\alpha}+\frac{\tau^{2 \alpha} \Gamma(\alpha+1)}{\Gamma(2 \alpha+1)}+\frac{\tau^{\alpha+1}}{\Gamma(\alpha+2)}+\frac{\tau^{\alpha}}{\Gamma(\alpha+1)}\right] \\
\xi\left[0-u_{0}(1, \tau)\right]=\xi\left[e\left(\tau^{\alpha}+\tau+1\right)-e\left(\tau+1+\tau^{\alpha}-\frac{\tau^{2 \alpha} \Gamma(\alpha+1)}{\Gamma(2 \alpha+1)}-\frac{\tau^{\alpha+1}}{\Gamma(\alpha+2)}-\frac{\tau^{\alpha}}{\Gamma(\alpha+1)}\right)\right]
\end{array}
$$


By putting Equations (21)-(23) in Equation (20) and simplifying, we get

$$
\begin{aligned}
& u_{0}^{*}(\xi, \tau)=e^{\xi}\left(1+\tau+\tau^{\alpha}-\frac{\tau^{2 \alpha} \Gamma(\alpha+1)}{\Gamma(2 \alpha+1)}-\frac{\tau^{\alpha+1}}{\Gamma(\alpha+2)}-\frac{\tau^{\alpha}}{\Gamma(\alpha+1)}\right) \\
& +(1-\xi+e \xi)\left(\frac{\tau^{2 \alpha} \Gamma(\alpha+1)}{\Gamma(2 \alpha+1)}+\frac{\tau^{\alpha+1}}{\Gamma(\alpha+2)}+\frac{\tau^{\alpha}}{\Gamma(\alpha+1)}\right) .
\end{aligned}
$$

From Equation (19), we have

$$
\begin{aligned}
& u_{1}(\xi, \tau)=L^{-1}\left(\frac{\partial^{2} u_{0}^{*}}{\partial \xi^{2}}\right) \\
& =L^{-1}\left(e^{\xi}\left(1+\tau+\tau^{\alpha}-\frac{\tau^{2 \alpha} \Gamma(\alpha+1)}{\Gamma(2 \alpha+1)}-\frac{\tau^{\alpha+1}}{\Gamma(\alpha+2)}-\frac{\tau^{\alpha}}{\Gamma(\alpha+1)}\right)\right), \\
& u_{1}(\xi, \tau)=e^{\xi}\left(\frac{\tau^{\alpha}}{\Gamma(\alpha+1)}+\frac{\tau^{\alpha+1}}{\Gamma(\alpha+2)}+\frac{\tau^{2 \alpha} \Gamma(\alpha+1)}{\Gamma(2 \alpha+1)}-\frac{\tau^{3 \alpha} \Gamma(\alpha+1)}{\Gamma(3 \alpha+1)}-\frac{\tau^{2 \alpha+1}}{\Gamma(2 \alpha+2)}-\frac{\tau^{2 \alpha}}{\Gamma(2 \alpha+1)}\right) . \\
& \text { For } n=1 \text {, Equation (17), we have } \\
& u_{1}^{*}(\xi, \tau)=u_{1}(\xi, \tau)+(1-\xi)\left[0-u_{1}(0, \tau)\right]+\xi\left[0-u_{1}(1, \tau)\right], \\
& u_{1}(\xi, \tau)=e^{\xi}\left(\frac{\tau^{\alpha}}{\Gamma(\alpha+1)}+\frac{\tau^{\alpha+1}}{\Gamma(\alpha+2)}+\frac{\tau^{2 \alpha} \Gamma(\alpha+1)}{\Gamma(2 \alpha+1)}-\frac{\tau^{3 \alpha} \Gamma(\alpha+1)}{\Gamma(3 \alpha+1)}-\frac{\tau^{2 \alpha+1}}{\Gamma(2 \alpha+2)}-\frac{\tau^{2 \alpha}}{\Gamma(2 \alpha+1)}\right) \\
& (1-\xi)\left[0-u_{1}(0, \tau)\right]=(1-\xi)\left[\tau^{\alpha}+\tau+1-\frac{\tau^{\alpha}}{\Gamma(\alpha+1)}-\frac{\tau^{\alpha+1}}{\Gamma(\alpha+2)}-\frac{\tau^{2 \alpha} \Gamma(\alpha+1)}{\Gamma(2 \alpha+1)}\right. \\
& \left.+\frac{\tau^{3 \alpha} \Gamma(\alpha+1)}{\Gamma(3 \alpha+1)}+\frac{\tau^{2 \alpha+1}}{\Gamma(2 \alpha+2)}+\frac{\tau^{2 \alpha}}{\Gamma(2 \alpha+1)}\right] \\
& \xi\left[0-u_{1}(1, \tau)\right]=\xi\left[e\left(\tau^{\alpha}+\tau+1-\frac{\tau^{\alpha}}{\Gamma(\alpha+1)}-\frac{\tau^{\alpha+1}}{\Gamma(\alpha+2)}-\frac{\tau^{2 \alpha} \Gamma(\alpha+1)}{\Gamma(2 \alpha+1)}+\frac{\tau^{3 \alpha} \Gamma(\alpha+1)}{\Gamma(3 \alpha+1)}+\frac{\tau^{2 \alpha+1}}{\Gamma(2 \alpha+2)}+\frac{\tau^{2 \alpha}}{\Gamma(2 \alpha+1)}\right)\right],
\end{aligned}
$$

By putting Equations (25)-(27) in Equation (24) and simplifying, we get

$$
\begin{aligned}
& u_{1}^{*}(\xi, \tau)=e^{\xi}\left(\frac{\tau^{\alpha}}{\Gamma(\alpha+1)}+\frac{\tau^{\alpha+1}}{\Gamma(\alpha+2)}+\frac{\tau^{2 \alpha} \Gamma(\alpha+1)}{\Gamma(2 \alpha+1)}-\frac{\tau^{3 \alpha} \Gamma(\alpha+1)}{\Gamma(3 \alpha+1)}-\frac{\tau^{2 \alpha+1}}{\Gamma(2 \alpha+2)}-\frac{\tau^{2 \alpha}}{\Gamma(2 \alpha+1)}\right) \\
& +(1-\xi+e \xi)\left[\tau^{\alpha}+\tau+1-\frac{\tau^{\alpha}}{\Gamma(\alpha+1)}-\frac{\tau^{\alpha+1}}{\Gamma(\alpha+2)}-\frac{\tau^{2 \alpha} \Gamma(\alpha+1)}{\Gamma(2 \alpha+1)}+\frac{\tau^{3 \alpha} \Gamma(\alpha+1)}{\Gamma(3 \alpha+1)}+\frac{\tau^{2 \alpha+1}}{\Gamma(2 \alpha+2)}+\frac{\tau^{2 \alpha}}{\Gamma(2 \alpha+1)}\right] .
\end{aligned}
$$

From Equation (19), we have

$$
\begin{gathered}
u_{2}(\xi, \tau)=L^{-1}\left(\frac{\partial^{2} u_{1}^{*}}{\partial \xi^{2}}\right), \\
=L^{-1}\left(e^{\xi}\left(\frac{\tau^{\alpha}}{\Gamma(\alpha+1)}+\frac{\tau^{\alpha+1}}{\Gamma(\alpha+2)}+\frac{\tau^{2 \alpha} \Gamma(\alpha+1)}{\Gamma(2 \alpha+1)}-\frac{\tau^{3 \alpha} \Gamma(\alpha+1)}{\Gamma(3 \alpha+1)}-\frac{\tau^{2 \alpha+1}}{\Gamma(2 \alpha+2)}-\frac{\tau^{2 \alpha}}{\Gamma(2 \alpha+1)}\right)\right), \\
u_{2}(\xi, \tau)=e^{\xi}\left(\frac{\tau^{2 \alpha+1}}{\Gamma(2 \alpha+2)}+\frac{\tau^{2 \alpha}}{\Gamma(2 \alpha+1)}+\frac{\tau^{3 \alpha} \Gamma(\alpha+1)}{\Gamma(3 \alpha+1)}-\frac{\tau^{4 \alpha} \Gamma(\alpha+1)}{\Gamma(4 \alpha+1)}-\frac{\tau^{3 \alpha+1}}{\Gamma(3 \alpha+2)}-\frac{\tau^{3 \alpha}}{\Gamma(3 \alpha+1)}\right) .
\end{gathered}
$$

For $n=2$, Equation (17) becomes,

$$
u_{2}^{*}(\xi, \tau)=u_{2}(\xi, \tau)+(1-\xi)\left[0-u_{2}(0, \tau)\right]+\xi\left[0-u_{2}(1, \tau)\right],
$$




$$
\begin{aligned}
& u_{2}(\xi, \tau)=e^{\xi}\left(\frac{\tau^{2 \alpha+1}}{\Gamma(2 \alpha+2)}+\right.\left.\frac{\tau^{2 \alpha}}{\Gamma(2 \alpha+1)}+\frac{\tau^{3 \alpha} \Gamma(\alpha+1)}{\Gamma(3 \alpha+1)}-\frac{\tau^{4 \alpha} \Gamma(\alpha+1)}{\Gamma(4 \alpha+1)}-\frac{\tau^{3 \alpha+1}}{\Gamma(3 \alpha+2)}-\frac{\tau^{3 \alpha}}{\Gamma(3 \alpha+1)}\right), \\
&(1-\xi)\left[0-u_{2}(0, \tau)\right]=(1-\xi)\left[\tau^{\alpha}+\tau+1-\frac{\tau^{2 \alpha+1}}{\Gamma(2 \alpha+2)}-\frac{\tau^{2 \alpha}}{\Gamma(2 \alpha+1)}-\frac{\tau^{3 \alpha} \Gamma(\alpha+1)}{\Gamma(3 \alpha+1)}+\right. \\
&\left.\frac{\tau^{4 \alpha} \Gamma(\alpha+1)}{\Gamma(4 \alpha+1)}+\frac{\tau^{3 \alpha+1}}{\Gamma(3 \alpha+2)}+\frac{\tau^{3 \alpha}}{\Gamma(3 \alpha+1)}\right], \\
& \xi\left[0-u_{2}(1, \tau)\right]=\xi\left[e\left(\tau^{\alpha}+\tau+1-\frac{\tau^{2 \alpha+1}}{\Gamma(2 \alpha+2)}-\frac{\tau^{2 \alpha}}{\Gamma(2 \alpha+1)}-\frac{\tau^{3 \alpha} \Gamma(\alpha+1)}{\Gamma(3 \alpha+1)}+\frac{\tau^{4 \alpha} \Gamma(\alpha+1)}{\Gamma(4 \alpha+1)}+\frac{\tau^{3 \alpha+1}}{\Gamma(3 \alpha+2)}+\frac{\tau^{3 \alpha}}{\Gamma(3 \alpha+1)}\right)\right],
\end{aligned}
$$

By putting Equations (29)-(31) in Equation (28) and simplifying ,we get

$$
\begin{aligned}
& u_{2}^{*}(\xi, \tau)=e^{\xi}\left(\frac{\tau^{2 \alpha+1}}{\Gamma(2 \alpha+2)}+\frac{\tau^{2 \alpha}}{\Gamma(2 \alpha+1)}+\frac{\tau^{3 \alpha} \Gamma(\alpha+1)}{\Gamma(3 \alpha+1)}-\frac{\tau^{4 \alpha} \Gamma(\alpha+1)}{\Gamma(4 \alpha+1)}-\frac{\tau^{3 \alpha+1}}{\Gamma(3 \alpha+2)}-\frac{\tau^{3 \alpha}}{\Gamma(3 \alpha+1)}\right) \\
& +(1-\xi+e \xi)\left(\tau^{\alpha}+\tau+1-\frac{\tau^{2 \alpha+1}}{\Gamma(2 \alpha+2)}-\frac{\tau^{2 \alpha}}{\Gamma(2 \alpha+1)}-\frac{\tau^{3 \alpha} \Gamma(\alpha+1)}{\Gamma(3 \alpha+1)}+\frac{\tau^{4 \alpha} \Gamma(\alpha+1)}{\Gamma(4 \alpha+1)}+\frac{\tau^{3 \alpha+1}}{\Gamma(3 \alpha+2)}+\frac{\tau^{3 \alpha}}{\Gamma(3 \alpha+1)}\right) .
\end{aligned}
$$

From Equation (19), we have

$$
\begin{gathered}
u_{3}(\xi, \tau)=L^{-1}\left(\frac{\partial^{2} u_{2}^{*}}{\partial \xi^{2}}\right), \\
=L^{-1}\left(e^{\xi}\left(\frac{\tau^{2 \alpha+1}}{\Gamma(2 \alpha+2)}+\frac{\tau^{2 \alpha}}{\Gamma(2 \alpha+1)}+\frac{\tau^{3 \alpha} \Gamma(\alpha+1)}{\Gamma(3 \alpha+1)}-\frac{\tau^{4 \alpha} \Gamma(\alpha+1)}{\Gamma(4 \alpha+1)}-\frac{\tau^{3 \alpha+1}}{\Gamma(3 \alpha+2)}-\frac{\tau^{3 \alpha}}{\Gamma(3 \alpha+1)}\right)\right), \\
u_{3}(\xi, \tau)=e^{\xi}\left(\frac{\tau^{3 \alpha+1}}{\Gamma(3 \alpha+2)}+\frac{\tau^{3 \alpha}}{\Gamma(3 \alpha+1)}+\frac{\tau^{4 \alpha} \Gamma(\alpha+1)}{\Gamma(4 \alpha+1)}-\frac{\tau^{5 \alpha} \Gamma(\alpha+1)}{\Gamma(5 \alpha+1)}-\frac{\tau^{4 \alpha+1}}{\Gamma(4 \alpha+2)}-\frac{\tau^{4 \alpha}}{\Gamma(4 \alpha+1)}\right) .
\end{gathered}
$$

Thus the ADM solution in the series form is,

$$
\begin{gathered}
u(\xi, \tau)=u_{0}(\xi, \tau)+u_{1}(\xi, \tau)+u_{2}(\xi, \tau)+u_{3}(\xi, \tau)+\ldots \\
=e^{\xi}+\tau\left(e^{\xi}\right)+e^{\xi}\left(\tau^{\alpha}-\frac{\tau^{2 \alpha} \Gamma(\alpha+1)}{\Gamma(2 \alpha+1)}-\frac{\tau^{\alpha+1}}{\Gamma(\alpha+2)}-\frac{\tau^{\alpha}}{\Gamma(\alpha+1)}\right) \\
+e^{\xi}\left(\frac{\tau^{\alpha}}{\Gamma(\alpha+1)}+\frac{\tau^{\alpha+1}}{\Gamma(\alpha+2)}+\frac{\tau^{2 \alpha} \Gamma(\alpha+1)}{\Gamma(2 \alpha+1)}-\frac{\tau^{3 \alpha} \Gamma(\alpha+1)}{\Gamma(3 \alpha+1)}-\frac{\tau^{2 \alpha+1}}{\Gamma(2 \alpha+2)}-\frac{\tau^{2 \alpha}}{\Gamma(2 \alpha+1)}\right) \\
+e^{\xi}\left(\frac{\tau^{2 \alpha+1}}{\Gamma(2 \alpha+2)}+\frac{\tau^{2 \alpha}}{\Gamma(2 \alpha+1)}+\frac{\tau^{3 \alpha} \Gamma(\alpha+1)}{\Gamma(3 \alpha+1)}-\frac{\tau^{4 \alpha} \Gamma(\alpha+1)}{\Gamma(4 \alpha+1)}-\frac{\tau^{3 \alpha+1}}{\Gamma(3 \alpha+2)}-\frac{\tau^{3 \alpha}}{\Gamma(3 \alpha+1)}\right) \\
+e^{\xi}\left(\frac{\tau^{3 \alpha+1}}{\Gamma(3 \alpha+2)}+\frac{\tau^{3 \alpha}}{\Gamma(3 \alpha+1)}+\frac{\tau^{4 \alpha} \Gamma(\alpha+1)}{\Gamma(4 \alpha+1)}-\frac{\tau^{5 \alpha} \Gamma(\alpha+1)}{\Gamma(5 \alpha+1)}-\frac{\tau^{4 \alpha+1}}{\Gamma(4 \alpha+2)}-\frac{\tau^{4 \alpha}}{\Gamma(4 \alpha+1)}\right)+\ldots
\end{gathered}
$$

In Figure 4a,b, the ADM-solutions of Example 2 are presented for different fractional order in 3D and 2D mode, respectively. The plots have shown the various dynamics of the physical phenomena represented by Example 2 for different values of $\alpha$. 


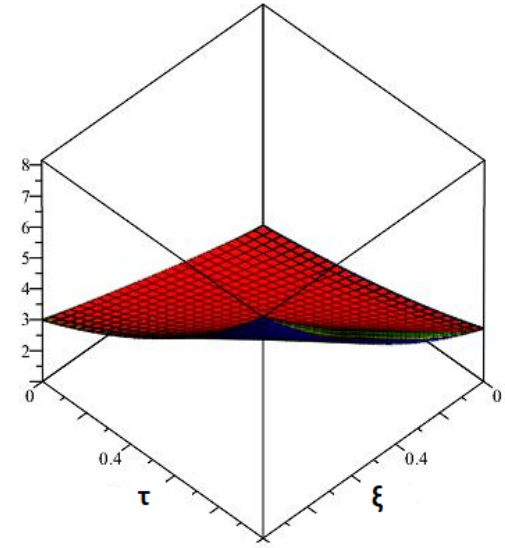

(a)

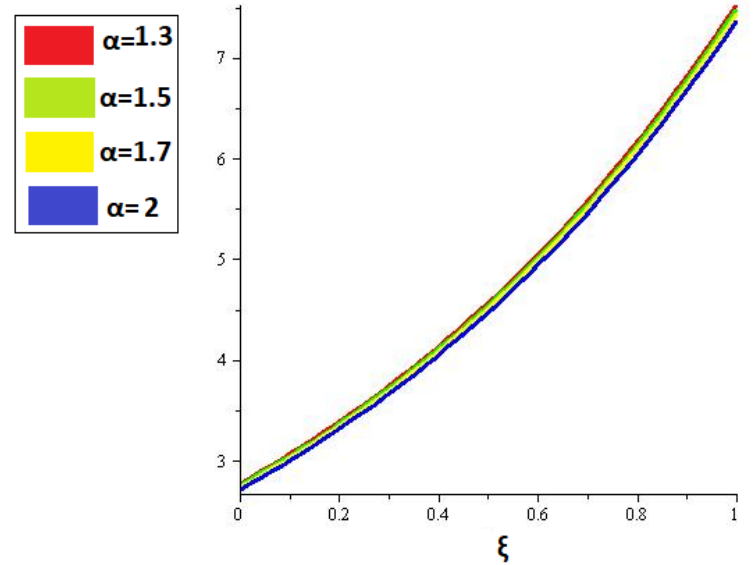

(b)

Figure 4. ADM solution plots of Example 2 (a) 3D and (b) 2D at different $\alpha$.

Figure 5 have shown the 3D Exact and ADM solutions of Example 2, respectively, at $\alpha=2$. It is observed that the exact and ADM solutions are in closed contact. Similarly, in Figure 6 the 2D exact and obtained results of Example 2 are plotted at $\alpha=2$. The Graphs have shown that the exact and obtained results are very closed and confirmed the validity of the present method.
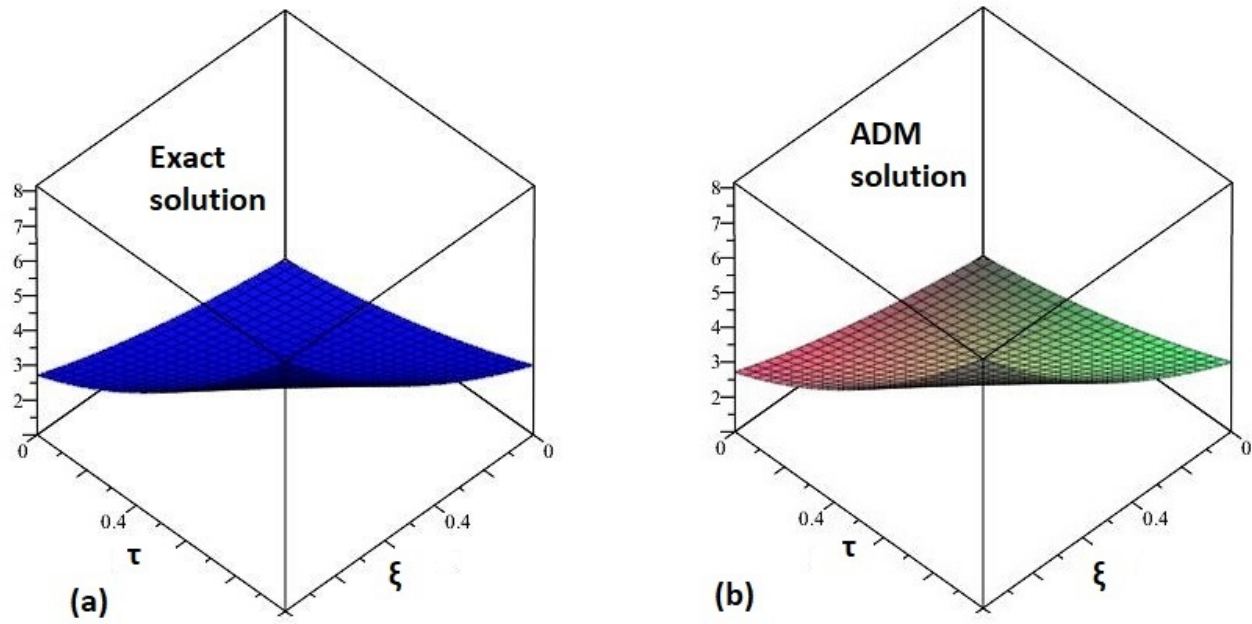

Figure 5. 3D solution plots (a) Exact and (b) ADM of Example 2 at $\alpha=2$.
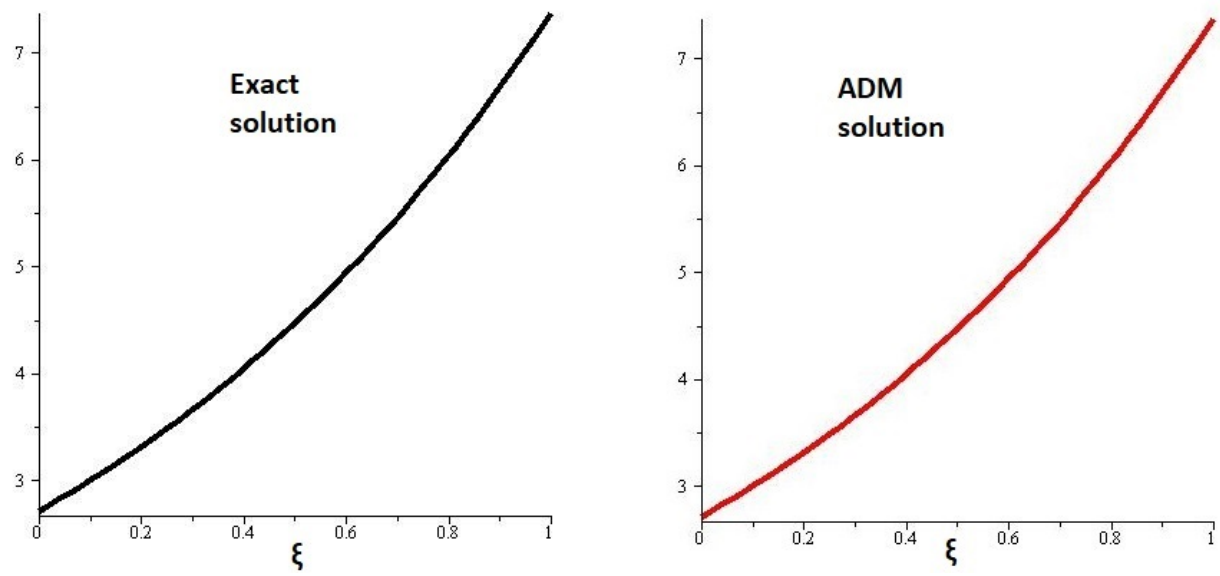

Figure 6. 2D Exact and ADM solution plot of Example 2 at $\alpha=2$. 


\subsection{Example 3}

Consider the FDWE of the form [70]

$$
D_{\tau}^{\alpha} u(\xi, \tau)=u_{\xi \xi}(\xi, \tau)+\sin (\xi)\left[\Gamma(2+\alpha) \tau+\tau^{1+\alpha}\right], \quad 0<\xi<\pi, 0<\tau \leq 1 .
$$

With the following initial and boundary conditions,

$$
\begin{aligned}
& u(\xi, 0)=0, \quad \partial_{t} u(\xi, 0)=0, \quad 0<\xi<1 \\
& u(0, \tau)=0, \quad u(\pi, \tau)=0, \quad 0<\tau \leq 1 .
\end{aligned}
$$

The problem has the Exact solution at $\alpha=2$ as follows,

$$
u(\xi, \tau)=\sin (\xi) \tau^{1+\alpha} .
$$

Applying the new technique based on ADM to Equation (32), we get the following result.

$$
u_{n}^{*}(\xi, \tau)=u_{n}(\xi, \tau)+(1-\xi)\left[0-u_{n}(0, \tau)\right]+\xi\left[0-u_{n}(\pi, \tau)\right] .
$$

where $n=0,1, \ldots$

Equation (32) can be written as,

$$
L u=u_{\xi \xi}(\xi, \tau)+\sin (\xi)\left[\Gamma(2+\alpha) \tau+\tau^{1+\alpha}\right],
$$

where $L=D_{\tau}^{\alpha}$ and $L^{-1}$ is defined as,

$$
L^{-1}(.)=I^{\alpha}(.) d \tau .
$$

Taking $L^{-1}$ on both sides of Equation (34), we get,

$$
u(\xi, \tau)=u(\xi, 0)+\tau\left(\partial_{t} u(\xi, 0)\right)+L^{-1}\left(u_{\xi \xi}(\xi, \tau)+\sin (\xi)\left[\Gamma(2+\alpha) \tau+\tau^{1+\alpha}\right]\right) .
$$

Using ADM solution, the initial approximation becomes,

$$
\begin{aligned}
u_{0}(\xi, \tau)=u(\xi, 0)+ & \tau\left(\partial_{t} u(\xi, 0)\right)+L^{-1}\left(\sin (\xi)\left[\Gamma(2+\alpha) \tau+\tau^{1+\alpha}\right]\right), \\
& =\sin (\xi)\left[\tau^{\alpha+1}+\frac{\tau^{1+2 \alpha}}{\Gamma(2+2 \alpha)}\right] \\
u_{0}(\xi, \tau) & =\sin (\xi) \tau^{\alpha+1}+\sin (\xi)\left[\frac{\tau^{1+2 \alpha}}{\Gamma(2+2 \alpha)}\right] .
\end{aligned}
$$

and use the new technique of initial approximation $u_{n}^{*}$ the iteration formula becomes

$$
u_{n+1}(\xi, \tau)=L^{-1}\left(\frac{\partial^{2} u_{n}^{*}}{\partial \xi^{2}}\right) .
$$

By putting the initial and boundary condition in Equation (33), for $n=0$

$$
\begin{gathered}
u_{0}^{*}(\xi, \tau)=u_{0}(\xi, \tau)+(1-\xi)\left[0-u_{0}(0, \tau)\right]+\xi\left[0-u_{0}(\pi, \tau)\right], \\
=\sin (\xi)\left[\tau^{\alpha+1}+\frac{\tau^{1+2 \alpha}}{\Gamma(2+2 \alpha)}\right]+(1-\xi)[0-0]+\xi[0-0], \\
=u_{0}^{*}(\xi, \tau)=\sin (\xi)\left[\tau^{\alpha+1}+\frac{\tau^{1+2 \alpha}}{\Gamma(2+2 \alpha)}\right] .
\end{gathered}
$$


From Equation (36), we have

$$
\begin{gathered}
u_{1}(\xi, \tau)=L^{-1}\left(\frac{\partial^{2} u_{0}^{*}}{\partial \xi^{2}}\right), \\
=L^{-1}\left(-\sin (\xi)\left[\tau^{\alpha+1}+\frac{\tau^{1+2 \alpha}}{\Gamma(2+2 \alpha)}\right]\right), \\
=-\sin (\xi)\left[\frac{\tau^{2 \alpha+1}}{\Gamma(2 \alpha+2)}+\frac{\tau^{1+3 \alpha}}{\Gamma(2+3 \alpha)}\right], \\
u_{1}(\xi, \tau)=-\sin (\xi)\left[\frac{\tau^{2 \alpha+1}}{\Gamma(2 \alpha+2)}\right]-\sin (\xi)\left[\frac{\tau^{1+3 \alpha}}{\Gamma(2+3 \alpha)}\right] .
\end{gathered}
$$

For $n=1$ Equation (33), we have

$$
\begin{gathered}
u_{1}^{*}(\xi, \tau)=u_{1}(\xi, \tau)+(1-\xi)\left[0-u_{1}(0, \tau)\right]+\xi\left[0-u_{1}(\pi, \tau)\right], \\
=-\sin (\xi)\left[\frac{\tau^{2 \alpha+1}}{\Gamma(2 \alpha+2)}+\frac{\tau^{1+3 \alpha}}{\Gamma(2+3 \alpha)}\right]+(1-\xi)[0-0]+\xi[0-0], \\
u_{1}^{*}(\xi, \tau)=-\sin (\xi)\left[\frac{\tau^{2 \alpha+1}}{\Gamma(2 \alpha+2)}+\frac{\tau^{1+3 \alpha}}{\Gamma(2+3 \alpha)}\right] .
\end{gathered}
$$

From Equation (36), we have

$$
\begin{gathered}
u_{2}(\xi, \tau)=L^{-1}\left(\frac{\partial^{2} u_{1}^{*}}{\partial \xi^{2}}\right), \\
=L^{-1}\left(\sin (\xi)\left[\frac{\tau^{2 \alpha+1}}{\Gamma(2 \alpha+2)}+\frac{\tau^{1+3 \alpha}}{\Gamma(2+3 \alpha)}\right]\right), \\
=\sin (\xi)\left[\frac{\tau^{3 \alpha+1}}{\Gamma(3 \alpha+2)}+\frac{\tau^{1+4 \alpha}}{\Gamma(2+4 \alpha)}\right], \\
u_{2}(\xi, \tau)=\sin (\xi)\left[\frac{\tau^{3 \alpha+1}}{\Gamma(3 \alpha+2)}\right]+\sin (\xi)\left[\frac{\tau^{1+4 \alpha}}{\Gamma(2+4 \alpha)}\right] .
\end{gathered}
$$

For $n=2$, Equation (33) becomes,

$$
\begin{gathered}
u_{2}^{*}(\xi, \tau)=u_{2}(\xi, \tau)+(1-\xi)\left[0-u_{2}(0, \tau)\right]+\xi\left[0-u_{2}(\pi, \tau)\right], \\
=\sin (\xi)\left[\frac{\tau^{3 \alpha+1}}{\Gamma(3 \alpha+2)}+\frac{\tau^{1+4 \alpha}}{\Gamma(2+4 \alpha)}\right]+(1-\xi)[0-0]+\xi[0-0], \\
=\left(\sin (\xi)\left[\frac{\tau^{3 \alpha+1}}{\Gamma(3 \alpha+2)}+\frac{\tau^{1+4 \alpha}}{\Gamma(2+4 \alpha)}\right]\right) . \\
u_{2}^{*}(\xi, \tau)=\sin (\xi)\left[\frac{\tau^{3 \alpha+1}}{\Gamma(3 \alpha+2)}+\frac{\tau^{1+4 \alpha}}{\Gamma(2+4 \alpha)}\right],
\end{gathered}
$$

From Equation (36), we have

$$
\begin{gathered}
u_{3}(\xi, \tau)=L^{-1}\left(\frac{\partial^{2} u_{2}^{*}}{\partial \xi^{2}}\right), \\
=L^{-1}\left(-\sin (\xi)\left[\frac{\tau^{3 \alpha+1}}{\Gamma(3 \alpha+2)}+\frac{\tau^{1+4 \alpha}}{\Gamma(2+4 \alpha)}\right]\right),
\end{gathered}
$$




$$
\begin{gathered}
=-\sin (\xi)\left[\frac{\tau^{4 \alpha+1}}{\Gamma(4 \alpha+2)}+\frac{\tau^{1+5 \alpha}}{\Gamma(2+5 \alpha)}\right], \\
u_{3}(\xi, \tau)=-\sin (\xi)\left[\frac{\tau^{4 \alpha+1}}{\Gamma(4 \alpha+2)}\right]-\sin (\xi)\left[\frac{\tau^{1+5 \alpha}}{\Gamma(2+5 \alpha)}\right] .
\end{gathered}
$$

Thus the ADM solution in the series form is,

$$
\begin{gathered}
u(\xi, \tau)=u_{0}(\xi, \tau)+u_{1}(\xi, \tau)+u_{2}(\xi, \tau)+u_{3}(\xi, \tau)+\ldots \\
=\sin (\xi)\left[\tau^{\alpha+1}\right]+\sin (\xi)\left[\frac{\tau^{1+2 \alpha}}{\Gamma(2+2 \alpha)}\right]-\sin (\xi)\left[\frac{\tau^{2 \alpha+1}}{\Gamma(2 \alpha+2)}\right]-\sin (\xi)\left[\frac{\tau^{1+3 \alpha}}{\Gamma(2+3 \alpha)}\right] \\
+\sin (\xi)\left[\frac{\tau^{3 \alpha+1}}{\Gamma(3 \alpha+2)}\right]+\sin (\xi)\left[\frac{\tau^{1+4 \alpha}}{\Gamma(2+4 \alpha)}\right]-\sin (\xi)\left[\frac{\tau^{4 \alpha+1}}{\Gamma(4 \alpha+2)}\right]+\sin (\xi)\left[\frac{\tau^{1+5 \alpha}}{\Gamma(2+5 \alpha)}\right]+\ldots
\end{gathered}
$$

Figure 7 represents the fractional order solutions of Example 3 with 2D and 3D geometry, respectively. The fractional order solutions confirm the validity of the proposed method to solve fractional initial and boundary value problems. The results are sophisticated and closely related to the exact solutions of the problems. Similarly, the plots given in Figure 8 indicates the 3D graphs of exact and obtained results of Example 3, respectively.

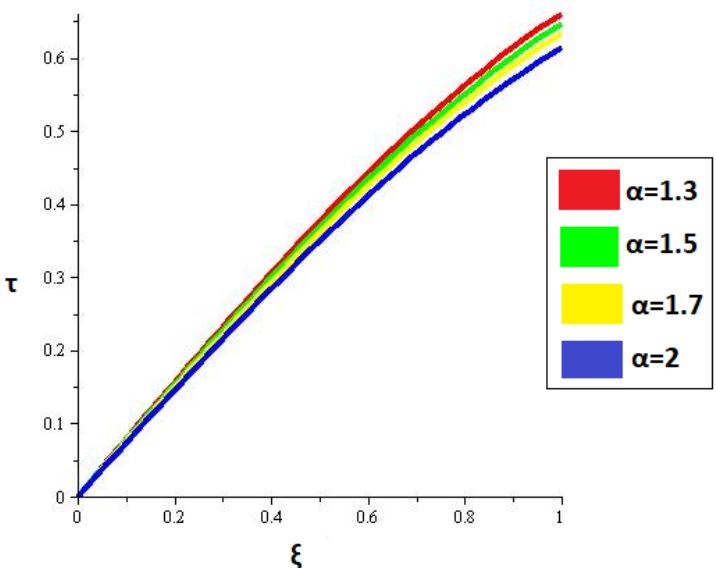

(a)

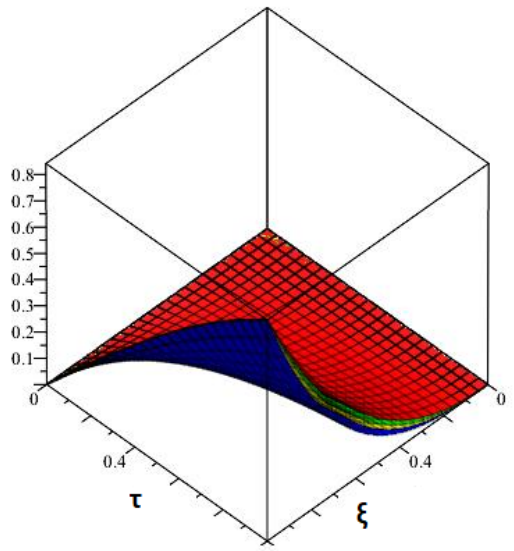

(b)

Figure 7. ADM solution plots (a) 2D and (b) 3D of Example 3 at different $\alpha$.
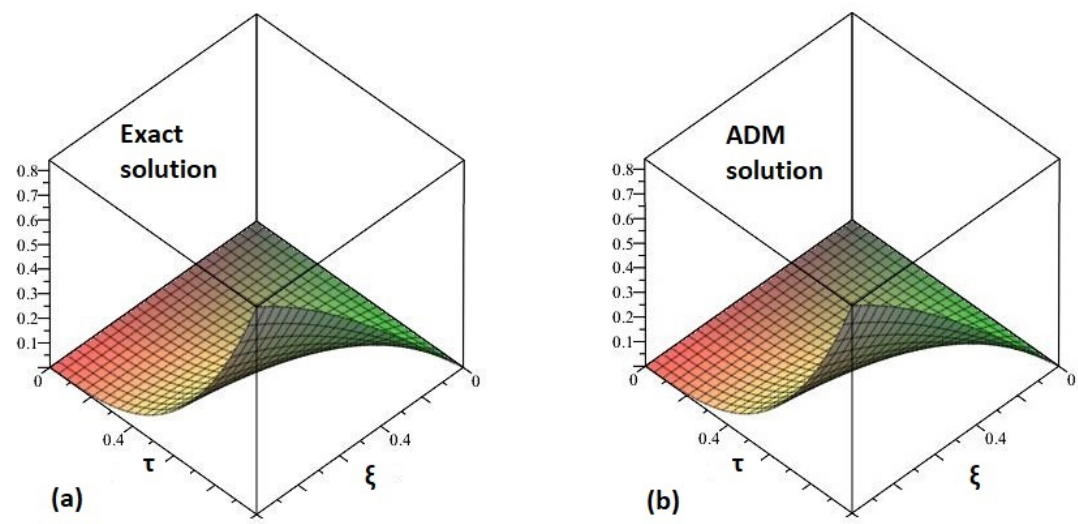

Figure 8. 3D solution plots (a) Exact and (b) ADM of Example 3 at $\alpha=2$. 
In Figure 9, the exact and ADM solution at $\alpha=2$ are plotted in 2D plots of example 3 . It is observed that the exact and derived results are in good contact and confirm the high accuracy of the proposed method.
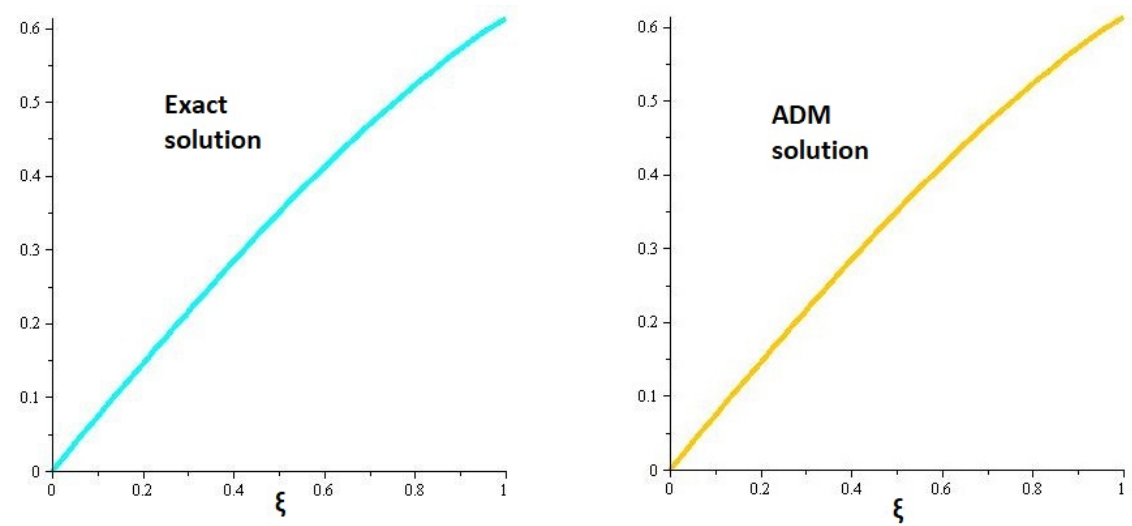

Figure 9. 2D Exact and ADM solution plots of Example 3 at $\alpha=2$.

In Table 1 , the solutions of Example 2 at $\alpha=1.3,1.5$ and 1.8 are calculated by using the new technique based on ADM. The derived results and accuracy of the method are listed in Table 1. It is confirmed that the accuracy of the proposed technique increases as $\alpha$ converges towards the integer order of the problem.

Table 1. Absolute error for the Example 2 at $t=0.8$.

\begin{tabular}{cccc}
\hline & $\mid$ Exact $-\boldsymbol{A D M} \mid$ & $\mid$ Exact $-\boldsymbol{A D M} \mid$ & $\mid$ Exact $\boldsymbol{A D M} \mid$ \\
\hline $\boldsymbol{\zeta}$ & $\boldsymbol{\alpha}=\mathbf{1 . 3}$ & $\boldsymbol{\alpha}=\mathbf{1 . 5}$ & $\boldsymbol{\alpha}=\mathbf{1 . 8}$ \\
\hline 0.1 & $2.469772 \times 10^{-3}$ & $4.68002 \times 10^{-4}$ & $3.2855 \times 10^{-5}$ \\
0.2 & $2.729520 \times 10^{-3}$ & $5.17222 \times 10^{-4}$ & $3.6311 \times 10^{-5}$ \\
0.3 & $3.016585 \times 10^{-3}$ & $5.71619 \times 10^{-4}$ & $4.0129 \times 10^{-5}$ \\
0.4 & $3.333843 \times 10^{-3}$ & $6.31737 \times 10^{-4}$ & $4.4351 \times 10^{-5}$ \\
0.5 & $3.684465 \times 10^{-3}$ & $6.98177 \times 10^{-4}$ & $4.9015 \times 10^{-5}$ \\
0.6 & $4.071964 \times 10^{-3}$ & $7.71604 \times 10^{-4}$ & $5.4170 \times 10^{-5}$ \\
0.7 & $4.500216 \times 10^{-3}$ & $8.52755 \times 10^{-4}$ & $5.9866 \times 10^{-5}$ \\
0.8 & $4.973509 \times 10^{-3}$ & $9.42440 \times 10^{-4}$ & $6.6163 \times 10^{-5}$ \\
0.9 & $5.496578 \times 10^{-3}$ & $1.041558 \times 10^{-3}$ & $7.3121 \times 10^{-5}$ \\
1 & $6.074659 \times 10^{-3}$ & $1.151100 \times 10^{-3}$ & $8.0812 \times 10^{-5}$
\end{tabular}

\section{Conclusions}

In this research article, fractional-order diffusion-wave equations with initial and boundary conditions are investigated analytically. A unique approach based on ADM is proposed for the solution of targeted problems in a very simple and effective manner. Fractional derivatives are defined in the Caputo sense for every example. Three examples of FDWEs are presented to confirm the validity of the suggested method. First, the modified ADM scheme is derived for general FPDEs and then implemented for some numerical examples of FDWEs. The solutions Graphs and Table are presented to show the best applicability of the current technique. The Graphs have confirmed that the present procedure is an effective tool for the solution of both integer and fractional order problems. The fractional solutions are handled in a very simple and sophisticated manner. Therefore the technique has a unique tendency to solve fractional PDEs with initial and boundary conditions and therefore it can be modified for the solution of other fractional initial-boundary value problems. According to our results, it is concluded that the present technique is good for FPDEs having both initial and boundary conditions, therefore the technique can be applied to other linear and non-linear FPDEs in future. 
Author Contributions: Conceptualization, H.K. and H.; methodology, H.; software, R.S.; validation, H.K., S.M. (Saima Mustafa) and S.M. (Saadia Masood); formal analysis, H.K.; investigation, H.; resources, R.S.; data curation, S.M. (Saima Mustafa); writing—original draft preparation, S.M. (Saima Mustafa); writing—review and editing, H.K.; visualization, H.; supervision, H.K.; project administration, $\mathrm{H}$.; funding acquisition, $\mathrm{H}$. All authors have read and agreed to the published version of the manuscript.

Funding: This research received no external funding.

Institutional Review Board Statement: Not applicable.

Informed Consent Statement: Not applicable.

Data Availability Statement: Not applicable.

Conflicts of Interest: The authors declare no conflict of interest.

\section{References}

1. Podlubny, I. Fractional Differential Equations: An Introduction to Fractional Derivatives, Fractional Differential Equations, to Methods of Their Solution and Some of Their Applications; Elsevier: Amsterdam, The Netherlands, 1998.

2. Miller, K.S.; Ross, B. An Introduction to the Fractional Calculus and Fractional Differential Equations; Wiley: Hoboken, NJ, USA, 1993.

3. Metzler, R.; Nonnenmacher, T.F. Space-and time-fractional diffusion and wave equations, fractional Fokker-Planck equations, and physical motivation. Chem. Phys. 2002, 284, 67-90. [CrossRef]

4. Metzler, R.; Klafter, J. The random walk's guide to anomalous diffusion: A fractional dynamics approach. Phys. Rep. 2000, 339, 1-77. [CrossRef]

5. Debnath, L.; Bhatta, D.D. Solutions to few linear fractional inhomogeneous partial differential equations in fluid mechanics. Fract. Calc. Appl. Anal. 2004, 7, 21-36.

6. He, J.H. Some applications of nonlinear fractional differential equations and their approximations. Bull. Sci. Technol. 1999, $15,86-90$.

7. Turut, V.; Güzel, N. On solving partial differential equations of fractional order by using the variational iteration method and multivariate Padé approximations. Eur. J. Pure Appl. Math. 2013, 6, 147-171.

8. Caputo, M. Linear models of dissipation whose Q is almost frequency independent-II. Geophys. J. Int. 1967, 13, 529-539. [CrossRef]

9. Sharma, M. Fractional integration and fractional differentiation of the M-series. Fract. Calc. Appl. Anal. 2008, 11, 187-191.

10. Rebenda, J.; Šmarda, Z. November. Numerical solution of fractional control problems via fractional differential transformation. In Proceedings of the 2017 European Conference on Electrical Engineering and Computer Science (EECS), Bern, Switzerland, 17-19 November 2017; pp. 107-111.

11. Lei, Y.; Wang, H.; Chen, X.; Yang, X.; You, Z.; Dong, S.; Gao, J. Shear property, high-temperature rheological performance and low-temperature flexibility of asphalt mastics modified with bio-oil. Constr. Build. Mater. 2018, 174, 30-37. [CrossRef]

12. Zhang, Z.Y. Symmetry determination and nonlinearization of a nonlinear time-fractional partial differential equation. Proc. $R$. Soc. 2020, 476, 20190564. [CrossRef]

13. Gorenflo, R.; Mainardi, F.; Moretti, D.; Paradisi, P. Time fractional diffusion: A discrete random walk approach. Nonlinear Dyn. 2002, 29, 129-143. [CrossRef]

14. Yang, Y.; Ma, Y.; Wang, L. Legendre polynomials operational matrix method for solving fractional partial differential equations with variable coefficients. Math. Probl. Eng. 2015, 2015, 915195. [CrossRef]

15. Karimi, K.; Rostamy, D.; Mohamadi, E. Solving fractional partial differential equations by an efficient new basis. Int. J. Appl. Math. Comput. 2013, 5, 6-21.

16. Magin, R.L. Fractional calculus in bioengineering, part 1. Crit. Rev. Biomed. Eng. 2004, 32. [CrossRef]

17. Tarasov, V.E. Fractional integro-differential equations for electromagnetic waves in dielectric media. Theor. Math. Phys. 2009, 158, 355-359. [CrossRef]

18. Magin, R.L. Fractional calculus in bioengineering, part 2. Crit. Rev. Biomed. Eng. 2004, 32. [CrossRef]

19. Odibat, Z.; Momani, S. A generalized differential transform method for linear partial differential equations of fractional order. Appl. Math. Lett. 2008, 21, 194-199. [CrossRef]

20. Yuanlu, L.I. Solving a nonlinear fractional differential equation using Chebyshev wavelets. Commun. Nonlinear Sci. Numer. Simul. 2010, 15, 2284-2292.

21. Hosseini, V.R.; Shivanian, E.; Chen, W. Local radial point interpolation (MLRPI) method for solving time fractional diffusion-wave equation with damping. J. Comput. Phys. 2016, 312, 307-332. [CrossRef]

22. Dehghan, M.; Abbaszadeh, M.; Mohebbi, A. Analysis of a meshless method for the time fractional diffusion-wave equation. Numer. Algorithms 2016, 73, 445-476. [CrossRef]

23. Jia, J.; Wang, H. A preconditioned fast finite volume scheme for a fractional differential equation discretized on a locally refined composite mesh. J. Comput. Phys. 2015, 299, 842-862. [CrossRef] 
24. Hejazi, H.; Moroney, T.; Liu, F. Stability and convergence of a finite volume method for the space fractional advection-dispersion equation. J. Comput. Appl. Math. 2014, 255, 684-697. [CrossRef]

25. Zhang, Y. A finite difference method for fractional partial differential equation. Appl. Math. Comput. 2009, 215, 524-529. [CrossRef]

26. Chen, S.; Liu, F.; Zhuang, P.; Anh, V. Finite difference approximations for the fractional Fokker-Planck equation. Appl. Math. Model. 2009, 33, 256-273. [CrossRef]

27. Jafari, H.; Seifi, S. Solving a system of nonlinear fractional partial differential equations using homotopy analysis method. Commun. Nonlinear Sci. Numer. Simul. 2009, 14, 1962-1969. [CrossRef]

28. Saravanan, A.; Magesh, N. A comparison between the reduced differential transform method and the Adomian decomposition method for the Newell-Whitehead-Segel equation. J. Egypt. Math. Soc. 2013, 21, 259-265. [CrossRef]

29. Lin, Y.; Xu, C. Finite difference/spectral approximations for the time-fractional diffusion equation. J. Comput. Phys. 2007, 225, 1533-1552. [CrossRef]

30. Zhang, S.; Zhang, H.Q. Fractional sub-equation method and its applications to nonlinear fractional PDEs. Phys. Lett. A 2011, 375, 1069-1073. [CrossRef]

31. Zheng, B. Exp-function method for solving fractional partial differential equations. Sci. World J. 2013, 2013, 465723. [CrossRef]

32. Ghosh, U.; Sengupta, S.; Sarkar, S.; Das, S. Analytic solution of linear fractional differential equation with Jumarie derivative in term of Mittag-Leffler function. arXiv 2015, arXiv:1505.06514.

33. Mainardi, F. Fractional Calculus And Waves in Linear Viscoelasticity: An Introduction To Mathematical Models; World Scientific: Singapore, 2010.

34. Nigmatullin, R.R. The realization of the generalized transfer equation in a medium with fractal geometry. Phys. Status Solidi B 1986, 133, 425-430. [CrossRef]

35. Meerschaert, M.M.; Benson, D.A.; Scheffler, H.P.; Baeumer, B. Stochastic solution of space-time fractional diffusion equations. Phys. Rev. E 2002, 65, 041103. [CrossRef]

36. Agrawal, O.P. Response of a diffusion-wave system subjected to deterministic and stochastic fields. Z. Angew. Math. Mech. 2003, 83, 265-274. [CrossRef]

37. Gorenflo, R.; Luchko, Y.; Mainardi, F. Wright functions as scale-invariant solutions of the diffusion-wave equation. J. Comput. Appl. Math. 2000, 118, 175-191. [CrossRef]

38. Agrawal, O.P. A general solution for the fourth-order fractional diffusion-wave equation. Fract. Calc. Appl. Anal. 2000, 3, 1-12.

39. Anh, V.V.; Leonenko, N.N. Harmonic analysis of random fractional diffusion-wave equations. Appl. Math. Comput. 2003, 141, 77-85. [CrossRef]

40. Luchko, Y. Fractional wave equation and damped waves. J. Math. Phys. 2013, 54, 031505. [CrossRef]

41. Luchko, Y. Wave-diffusion dualism of the neutral-fractional processes. J. Comput. Phys. 2015, 293, 40-52. [CrossRef]

42. Luchko, Y. Initial-boundary-value problems for the one-dimensional time-fractional diffusion equation. Fract. Calc. Appl. Anal. 2012, 15, 141-160. [CrossRef]

43. Sakamoto, K.; Yamamoto, M. Initial value/boundary value problems for fractional diffusion-wave equations and applications to some inverse problems. J. Math. Anal. Appl. 2011, 382, 426-447. [CrossRef]

44. Kong, F.; Zhu, Q. New fixed-time synchronization control of discontinuous inertial neural networks via indefinite LyapunovKrasovskii functional method. Int. J. Robust Nonlinear Control 2021, 31, 471-495. [CrossRef]

45. Jin, C.; Liu, M. A new modification of Adomian decomposition method for solving a kind of evolution equation. Appl. Math. Comput. 2005, 169, 953-962. [CrossRef]

46. Pue-On, P.; Viriyapong, N. Modified Adomian decomposition method for solving particular third-order ordinary differential equations. Appl. Math. Sci. 2012, 6, 1463-1469.

47. Hasan, Y.Q.; Zhu, L.M. Modified Adomian decomposition method for singular initial value problems in the second-order ordinary differential equations. Surv. Math. Appl. 2008, 3, 183-193.

48. Jafari, H.; Daftardar-Gejji, V. Revised Adomian decomposition method for solving systems of ordinary and fractional differential equations. Appl. Math. Comput. 2006, 181, 598-608. [CrossRef]

49. Khan, H.; Khan, A.; Kumam, P.; Baleanu, D.; Arif, M. An approximate analytical solution of the Navier-Stokes equations within Caputo operator and Elzaki transform decomposition method. Adv. Differ. Equ. 2020, 2020, 1-23.

50. Shah, R.; Khan, H.; Arif, M.; Kumam, P. Application of Laplace-Adomian decomposition method for the analytical solution of third-order dispersive fractional partial differential equations. Entropy 2019, 21, 335. [CrossRef]

51. Mahmood, S.; Shah, R.; Arif, M. Laplace Adomian decomposition method for multi dimensional time fractional model of Navier-Stokes equation. Symmetry 2019, 11, 149. [CrossRef]

52. Shah, R.; Khan, H.; Mustafa, S.; Kumam, P.; Arif, M. Analytical Solutions of Fractional-Order Diffusion Equations by Natural Transform Decomposition Method. Entropy 2019, 21, 557. [CrossRef]

53. Khan, H.; Shah, R.; Baleanu, D.; Kumam, P.; Arif, M. Analytical Solution of Fractional-Order Hyperbolic Telegraph Equation, Using Natural Transform Decomposition Method. Electronics 2019, 8, 1015. [CrossRef]

54. Shah, R.; Farooq, U.; Khan, H.; Baleanu, D.; Kumam, P.; Arif, M. Fractional view analysis of third order Kortewege-De Vries equations, using a new analytical technique. Front. Phys. 2020, 7, 244. [CrossRef]

55. Ali, I.; Khan, H.; Farooq, U.; Baleanu, D.; Kumam, P.; Arif, M. An Approximate-Analytical Solution to Analyze Fractional View of Telegraph Equations. IEEE Access 2020, 8, 25638-25649. [CrossRef] 
56. Momani, S.; Odibat, Z. Analytical solution of a time-fractional Navier-Stokes equation by Adomian decomposition method. Appl. Math. Comput. 2006, 177, 488-494. [CrossRef]

57. Bildik, N.; Konuralp, A. Two-dimensional differential transform method, Adomian's decomposition method, and variational iteration method for partial differential equations. Int. J. Comput. Math. 2006, 83, 973-987. [CrossRef]

58. Oda, A.H.; Al Q.; Lafta, H.F. Modified algorithm to compute Adomian's polynomial for solving non-linear systems of partial differential equations. Int. Contemp. Math. Sci. 2010, 5, 2505-2521.

59. Hashim, I. Adomian decomposition method for solving BVPs for fourth-order integro-differential equations. J. Comput. Appl. Math. 2006, 193, 658-664. [CrossRef]

60. Wazwaz, A.M. The combined Laplace transform-Adomian decomposition method for handling nonlinear Volterra integro-differential equations. Appl. Math. Comput. 2010, 216, 1304-1309. [CrossRef]

61. Abdou, M.A. Solitary Solutions of Nonlinear Differential-difference Equations via Adomain Decomposition Method. Int. J. Nonlinear Sci. 2011, 12, 29-35.

62. Wang, Z.; Zou, L.; Zong, Z. Adomian decomposition and Padé approximate for solving differential-difference equation. Appl. Math. Comput. 2011, 218, 1371-1378. [CrossRef]

63. Hosseini, M.M. Adomian decomposition method for solution of nonlinear differential algebraic equations. Appl. Math. Comput. 2006, 181, 1737-1744. [CrossRef]

64. Hosseini, M.M. Adomian decomposition method for solution of differential-algebraic equations. J. Comput. Appl. Math. 2006, 197, 495-501. [CrossRef]

65. Ali, E.J. A new technique of initial boundary value problems using Adomian decomposition method. Int. Math. Forum 2012, 7, 799-814.

66. Ali, E.J. New Treatment of Initial Boundary Problems for Fourth-Order Parabolic Partial Differential Equations Using Variational Iteration Method. Int. J. Contemp. Math. Sci. 2011, 6, 2367-2376.

67. Ali, E.J. New treatment of the solution of initial boundary value problems by using variational iteration method. Basrah J. Sci. 2012, 30, 57-74.

68. Ahmed, A.A.I.; Ali, E.J.; Jassim, A.M. A new procedure of initial boundary value problems using homotopy perturbation method. J. Kufa Math. Comput. 2013, 1, 54-62.

69. Alia, A.; Shaha, K.; Lib, Y.; Khana, R.A. Numerical treatment of coupled system of fractional order partial differential equations. J. Math. Comput. Sci. 2019, 19, 74-85. [CrossRef]

70. Chen, A.; Li, C. Numerical solution of fractional diffusion-wave equation. Numer. Funct. Anal. Optim. 2016, 37, 19-39. [CrossRef] 